\title{
Molecular model of biaxial ordering in nematic liquid crystals composed of flat molecules with four mesogenic groups
}

\author{
M. V. Gorkunov, ${ }^{1,2}$ M. A. Osipov, ${ }^{3}$ A. Kocot, ${ }^{1,4}$ and J. K. Vij ${ }^{1}$ \\ ${ }^{1}$ Department of Electronic and Electrical Engineering, Trinity College Dublin, Dublin 2, Ireland \\ ${ }^{2}$ A. V. Shubnikov Institute of Crystallography, Russian Academy of Sciences, 119333 Moscow, Russia \\ ${ }^{3}$ Department of Mathematics, University of Strathclyde, Glasgow G1 1XH, United Kingdom \\ ${ }^{4}$ Institute of Physics, University of Silesia, Katowice, Poland \\ (Received 19 January 2010; revised manuscript received 17 May 2010; published 11 June 2010)
}

\begin{abstract}
Relative stability of uniaxial and biaxial nematic phases is analyzed in a model nematic liquid crystal composed of flat molecules of $C_{2 h}$ symmetry with four mesogenic groups rigidly linked to the same center. The generalized effective quadrupole mean-field potential is proposed and its constants are evaluated numerically for the pair intermolecular potential based on Gay-Berne interaction between mesogenic groups. The dependencies of the constants on molecular shape parameters are systematically analyzed. Order parameters of the uniaxial and biaxial nematic phases are evaluated by direct minimization of the free energy at different temperatures. The corresponding phase diagrams are obtained enabling one to study the effects of molecular model parameters on the stability regions of uniaxial and biaxial phases. The results are used to clarify the nature of experimentally observed biaxial ordering in nematic liquid crystals composed of tetrapode molecules with the same symmetry.
\end{abstract}

DOI: 10.1103/PhysRevE.81.061702

PACS number(s): 64.70.mf, 61.30.Cz, 42.70.Df

\section{INTRODUCTION}

Liquid crystals are composed of strongly anisotropic molecules which are generally biaxial. At the same time, the simplest liquid-crystal phases, i.e., conventional nematic and smectic A ones, are macroscopically uniaxial. In uniaxial phases, both long and short molecular axes are distributed symmetrically around a unique macroscopic axis specified by the director $\mathbf{n}$. At lower temperatures many liquid-crystal materials exhibit tilted smectic phases in which the director is not parallel to the smectic layer normal. Tilted smectic phases are intrinsically biaxial, and in these systems the molecular long axes are mainly ordered along the director while short molecular axes are ordered along a direction perpendicular to the tilt plane.

It should be noted, however, that in tilted smectic phases the biaxial ordering of short molecular axes does not appear in a self-consistent way but is induced by the tilt of the director. From this point of view one of the greatest challenges in the field of liquid crystals is related to the biaxial nematic phase in which molecular long and short axes are ordered along the two macroscopic orthogonal directions specified by the primary director $\mathbf{n}$ and the secondary director $\mathbf{m}$. The biaxial nematic phase is characterized by three different principal refractive indices. Apart from their fundamental significance, biaxial nematic materials are also strategically important from the technological point of view because they have a number of advantages over both conventional nematics and tilted smectics including a possibility of ultrafast electro-optical and all-optical switching of the second director.

The thermotropic biaxial nematic phase was predicted theoretically 40 years ago by Freiser [1]. In 1980, Yu and Saupe discovered the biaxial nematic phase for a lyotropic liquid crystal composed of relatively large micelles [2]. Many compounds have since been claimed to form a ther- motropic biaxial nematic phase but there were no sufficient experimental evidences. A potential existence of the biaxial nematic phase has been supported by computer simulations including the studies of lattice models [3,4], athermal systems of hard biaxial ellipsoids [5,6] and soft biaxial particles interacting via the generalized Gay-Berne potential [7] and $\mathrm{V}$-shaped multisite interaction models for bent-core molecules $[8,9]$. Recently the results of computer simulations of biaxial nematics have been summarized in a review [10].

In recent years strong experimental evidence in favor of the existence of the thermotropic biaxial nematic phase has finally been presented for two different materials. The biaxial ordering has first been found in the nematic phase of bentcore mesogens based on a mesogenic oxadiazole core $[11,12]$. The biaxial phase has been characterized by $\mathrm{x}$-ray scattering [13], conoscopy, and deuterium NMR [14] and supported by atomistic computer simulations [9]. Very recently the biaxial nematic ordering in bent-core liquid crystals has also been studied using polarized Raman scattering [15], and several biaxial order parameters have been determined. The second biaxial nematic material is composed of tetrapode molecules in which four mesogenic groups are linked by flexible spacers to the central atom [16-18]. Polarized infrared spectroscopy has been used to determine the four orientational order parameters of this biaxial nematic [16]. The existence of the biaxial ordering has later been supported by NMR using a deuterated calamitic mesogen as a spin probe [19].

Pioneering works in the molecular theory of biaxial nematic liquid crystals have been published by Fraser [1] and Straley [20]. In particular, Straley has proposed a general model quadrupole interaction potential composed of second rank molecular tensors. This model potential has been used in the majority of molecular theories developed later by different authors. In the molecular theory based on the quadrupole potential, the system undergoes a transition from the 
isotropic to the uniaxial calamitic $N_{U}$ or uniaxial discotic $N_{D}$ nematic phase depending on the sign of molecular biaxiality [21]. This first-order transition is followed by the secondorder transition from the uniaxial to the biaxial nematic phase. The phase diagram of such a system is also characterized by the Landau triple point where the material undergoes a direct transition from the isotropic to the biaxial nematic phase [20-23]. Recently Virga, Sonnet, and Bisi with coauthors have presented a generalized mean-field phase diagram for biaxial nematics [24-26] which contains both tricritical and triple points. Four orientational order parameters of the biaxial nematic phase have recently been calculated numerically using the same model [27].

A drawback of all molecular theories based on a model interaction potential is related to the difficulties in establishing a correlation between the molecular structure and the values of model parameters. Only a molecular model based on dispersion interaction between biaxial molecules has been used to express the coefficients of the model potential in terms of the components of molecular polarizability [21]. No attention has been paid so far to a relationship between the symmetry of constituent biaxial molecules and the relative strength of the biaxial part of the interaction potential, which determines the stability of the biaxial nematic phase. However, such a relationship is very important. For example, several attempts to tailor real disklike molecules with strong biaxiality have not led to a biaxial nematic phase (see, for example, [28]). At the same time, biaxial ordering has been discovered in mesogens of rather unusual structure including bent-core molecules and tetrapodes containing four mesogenic groups linked to the same center.

In this paper we consider a simple model of a rigid biaxial molecule composed of four uniaxial mesogenic groups interacting via a Gay-Berne potential. The biaxiality of such a molecule is determined by the anisotropy of its geometrical frame and by the angle between the mesogenic groups and the axes of that frame. Taking into account the interactions between all pairs of mesogenic groups within any two interacting molecules we expand the total intermolecular interaction potential in orthogonal functions and calculate all parameters of the corresponding effective quadrupole potential numerically as functions of the model parameters of the molecular structure. We then employ the molecular-field theory of biaxial nematics to investigate the phase diagram of the system and to calculate numerically the order parameters of the uniaxial and biaxial nematic phases. Although the studied rigid molecules cannot be considered as a truly realistic model for the real flexible tetrapode molecules, they possess the same point symmetry as the averaged conformation of a tetrapode. This enables applying our results to shed some light on the origin of the biaxial ordering observed in the real liquid crystals.

\section{PAIR INTERMOLECULAR INTERACTION POTENTIAL}

The orientation of a rigid biaxial molecule can be specified by the unit vectors $\mathbf{a}$ and $\mathbf{b}$ in the direction of the primary ("long") and secondary ("short") molecular axes, re-

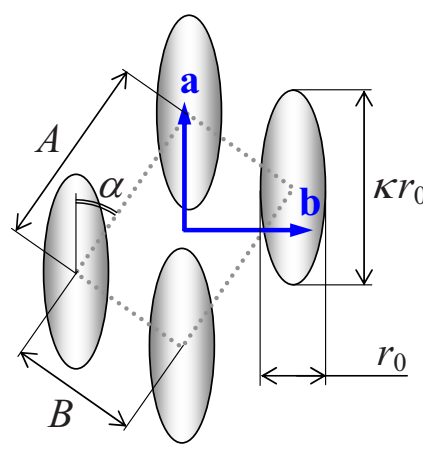

FIG. 1. (Color online) A model of a biaxial molecule of the $C_{2 h}$ symmetry with molecular axes $\mathbf{a}$ and $\mathbf{b}$ composed of four mesogenic groups.

spectively. The third molecular axis is then specified by the unit vector $\mathbf{c}$ which is orthogonal to both $\mathbf{a}$ and $\mathbf{b}$. In the biaxial nematic phase there exist three mutually orthogonal macroscopic directions specified by the primary director $\mathbf{n}$ and the secondary directors $\mathbf{m}$ and $\mathbf{h}$. The unit vectors $\mathbf{a}, \mathbf{b}$ and $\mathbf{c}$, which form the molecular frame, can be expressed in the macroscopic $\{\mathbf{n}, \mathbf{m}, \mathbf{h}\}$ frame in the following way:

$$
\mathbf{a}=\mathbf{n} \cos \gamma+\mathbf{m} \sin \gamma \cos \phi+\mathbf{h} \sin \gamma \sin \phi
$$

and

$$
\begin{aligned}
\mathbf{b}= & -\mathbf{n} \sin \gamma \cos \psi+\mathbf{m}(\cos \gamma \cos \phi \cos \psi-\sin \phi \sin \psi) \\
& +\mathbf{h}(\cos \gamma \sin \phi \cos \psi+\cos \phi \sin \psi)
\end{aligned}
$$

and

$$
\begin{aligned}
\mathbf{c}= & \mathbf{n} \sin \gamma \sin \psi-\mathbf{m}(\cos \gamma \cos \phi \sin \psi+\sin \phi \cos \psi) \\
& +\mathbf{h}(\cos \phi \cos \psi-\cos \gamma \sin \phi \sin \psi),
\end{aligned}
$$

where $\gamma, \phi$, and $\psi$ are the corresponding angles as follows: the angle $\gamma$ is the polar angle between the primary axis a and the primary director $\mathbf{n}$, the angle $\phi$ is the azimuthal angle which specifies the orientation of the projection of the axis a on the $(\mathbf{m}, \mathbf{h})$ plane, and $\psi$ specifies the angle between the short axis $\mathbf{b}$ and the $(\mathbf{a}, \mathbf{n})$ plane. A pair interaction potential for two biaxial molecules " 1 " and " 2 " is generally a function of all molecular orientations and the intermolecular vector $\mathbf{r}=\mathbf{r}_{12}$, i.e., $U(1,2)=u\left(\mathbf{r}, \mathbf{a}_{1}, \mathbf{a}_{2}, \mathbf{b}_{1}, \mathbf{b}_{2}\right)$.

We consider the model of a rigid flat biaxial molecule of the $C_{2 h}$ symmetry composed of four aligned mesogenic groups (see Fig. 1). The groups are parallel, lie in the same plane, and their centers are located on the corners of a rectangle with sides $A$ and $B$. The mesogenic groups are tilted by an angle $\alpha$ with respect to the long axis of the rectangle.

The main part of the intermolecular interaction for such molecules arises from the cross-interaction of pairs of mesogenic groups belonging to the two molecules. This interaction potential can be written as a sum of 16 similar terms:

$$
u\left(\mathbf{r}, \mathbf{a}_{1}, \mathbf{a}_{2}, \mathbf{b}_{1}, \mathbf{b}_{2}\right)=\sum_{\eta, \xi=1}^{4} V\left(\mathbf{a}_{1}, \mathbf{r}_{\eta \xi}, \mathbf{a}_{2}\right),
$$

where $\mathbf{r}_{\eta \xi}$ is the vector between the $\eta$ th group of the first molecule and the $\xi$ th group of the second one, and the pri- 
mary molecular axes $\mathbf{a}_{1,2}$ of the molecules are parallel to the long axes of mesogenic groups in the corresponding molecule.

Anisotropic interaction between mesogenic groups is often modeled by the Gay-Berne (GB) interaction potential $[29,30]$ which is popular for its relative simplicity, smoothness, and capability of reproducing orientational order of anisotropic liquids [30-33]. We use the following expression for the GB potential $V_{\mathrm{GB}}(1,2)$ to describe the pair interaction of the mesogenic groups:

$$
\begin{aligned}
V_{\mathrm{GB}}\left(\mathbf{a}_{1}, \mathbf{R}, \mathbf{a}_{2}\right)= & 4 \varepsilon\left(\mathbf{a}_{1}, \hat{\mathbf{r}}, \mathbf{a}_{2}\right) \times\left\{\left[\frac{R}{r_{0}}-\sigma\left(\mathbf{a}_{1}, \hat{\mathbf{r}}, \mathbf{a}_{2}\right)+1\right]^{-12}\right. \\
& \left.-\left[\frac{R}{r_{0}}-\sigma\left(\mathbf{a}_{1}, \hat{\mathbf{r}}, \mathbf{a}_{2}\right)+1\right]^{-6}\right\}
\end{aligned}
$$

where the orientation dependent range is

$$
\begin{aligned}
\sigma\left(\mathbf{a}_{1}, \hat{\mathbf{r}}, \mathbf{a}_{2}\right)= & \left\{1-\frac{\chi}{2}\left[\frac{\left(\hat{\mathbf{r}} \cdot \mathbf{a}_{1}+\hat{\mathbf{r}} \cdot \mathbf{a}_{2}\right)^{2}}{1+\chi \mathbf{a}_{1} \cdot \mathbf{a}_{2}}\right.\right. \\
& \left.\left.+\frac{\left(\hat{\mathbf{r}} \cdot \mathbf{a}_{1}-\hat{\mathbf{r}} \cdot \mathbf{a}_{2}\right)^{2}}{1-\chi \mathbf{a}_{1} \cdot \mathbf{a}_{2}}\right]\right\}^{-1 / 2}
\end{aligned}
$$

and the anisotropic interaction strength is expressed as

$$
\begin{aligned}
\varepsilon\left(\mathbf{a}_{1}, \hat{\mathbf{r}}, \mathbf{a}_{2}\right) & \\
= & \varepsilon_{0}\left[1-\chi^{2}\left(\mathbf{a}_{1} \cdot \mathbf{a}_{2}\right)^{2}\right]^{-1 / 2} \\
& \times\left\{1-\frac{\chi^{\prime}}{2}\left[\frac{\left(\hat{\mathbf{r}} \cdot \mathbf{a}_{1}+\hat{\mathbf{r}} \cdot \mathbf{a}_{2}\right)^{2}}{1+\chi^{\prime} \mathbf{a}_{1} \cdot \mathbf{a}_{2}}+\frac{\left(\hat{\mathbf{r}} \cdot \mathbf{a}_{1}-\hat{\mathbf{r}} \cdot \mathbf{a}_{2}\right)^{2}}{1-\chi^{\prime} \mathbf{a}_{1} \cdot \mathbf{a}_{2}}\right]\right\}^{2} .
\end{aligned}
$$

Here $\hat{\mathbf{r}}=\mathbf{R} / R, r_{0}$ is the breadth of the molecule and the constants $\chi=\left(\kappa^{2}-1\right) /\left(\kappa^{2}+1\right)$ and $\chi^{\prime}=\left(\kappa^{\prime 1 / 2}-1\right) /\left(\kappa^{\prime 1 / 2}+1\right)$ are determined by the relative elongation of the mesogenic groups $\kappa$ as well as by the ratio $\kappa^{\prime}$ of the energy well depths for side to side and end to end mesogenic group orientations.

To take into account the hard-rod nature of the mesogenic groups, which cannot penetrate each other, we multiply the GB potential by 16 step functions of steric cutoff which take the form $\Theta\left[r_{\eta \xi}-r_{0} \sigma\left(\mathbf{a}_{1}, \hat{\mathbf{r}}, \mathbf{a}_{2}\right)\right]$. Thus we consider only those pair molecular configurations when none of the groups intersect.

\section{MOLECULAR FIELD THEORY OF NEMATICS WITH BIAXIAL ORDERING}

\section{A. Order parameters}

Since the pioneering work of Straley [20] the following two tensor order parameters: $Q_{i j}=\left\langle a_{i} a_{j}-(1 / 3) \delta_{i j}\right\rangle$ and $B_{i j}$ $=\left\langle b_{i} b_{j}-c_{i} c_{j}\right\rangle$, where $\langle\ldots\rangle$ denotes the statistical average, have been used to describe the thermodynamic properties of biaxial nematics. However, as has been shown recently [34], for the molecules of $C_{2 h}$ symmetry with the symmetry axis parallel to the axis $\mathbf{c}$ it is possible to introduce the third independent molecular invariant $a_{i} b_{j}$ which is invariant under all symmetry transformations of such a molecule and which is orthogonal to both invariants $a_{i} a_{j}-(1 / 3) \delta_{i j}$ and $b_{i} b_{j}-c_{i} c_{j}$ used in the existing theories. This new molecular invariant gives rise to the new tensor order parameter $\Gamma_{i j}$ $=\left\langle a_{i} b_{j}+b_{i} a_{j}\right\rangle$. The statistical average of the antisymmetric part of the molecular tensor $\Omega_{i j}=\left\langle a_{i} b_{j}-b_{i} a_{j}\right\rangle$ is equivalent to the macroscopic pseudovector $M_{i}=\epsilon_{i j k} \Omega_{j k}$ where $\epsilon_{i j k}$ is the absolute antisymmetric Levy-Civita tensor.

The tensor order parameters $\mathbf{Q}, \mathbf{B}$, and $\boldsymbol{\Gamma}$ are second rank symmetric tensors and thus they can be diagonalized. However, the main axes of all these tensors will coincide only in the high-symmetry orthogonal biaxial nematic phase which is characterized by three mutually perpendicular mirror planes. The unit normals to these planes define three directors $\{\mathbf{n}, \mathbf{m}, \mathbf{h}\}$ of the biaxial nematic phase. This highsymmetry biaxial nematic phase is assumed in the existing molecular theory of biaxial ordering in nematics and in all experimental papers. One notes that a low symmetry biaxial nematic phases may also exist in principle [35], but at present there is no direct evidence on the precise symmetry of any of the biaxial nematic phases studied experimentally. Taking into account that the main aim of this paper is the study of the tendency to exhibit the biaxial order depending on the molecular structure, we follow the assumption of the previous authors for simplicity and consider the highsymmetry biaxial phase.

In the orthogonal biaxial phase the three tensor order parameters can be expressed in the common diagonal frame $\{\mathbf{n}, \mathbf{m}, \mathbf{h}\}$ as

$$
\begin{gathered}
Q_{i j}=S\left(n_{i} n_{j}-\frac{1}{3} \delta_{i j}\right)+\frac{1}{2} P\left(m_{i} m_{j}-h_{i} h_{j}\right), \\
B_{i j}=D\left(n_{i} n_{j}-\frac{1}{3} \delta_{i j}\right)+C\left(m_{i} m_{j}-h_{i} h_{j}\right), \\
\Gamma_{i j}=G\left(n_{i} n_{j}-\frac{1}{3} \delta_{i j}\right)+H\left(m_{i} m_{j}-h_{i} h_{j}\right),
\end{gathered}
$$

where the six scalar order parameters are expressed in terms of the following statistical averages:

$$
\begin{gathered}
S=\left\langle P_{2}(\cos \gamma)\right\rangle, \\
P=\left\langle\sin ^{2} \gamma \cos 2 \phi\right\rangle, \\
D=\frac{3}{2}\left\langle\sin ^{2} \gamma \cos 2 \psi\right\rangle,
\end{gathered}
$$

$$
C=\frac{1}{2}\left\langle\cos 2 \phi \cos 2 \psi\left(1+\cos ^{2} \gamma\right)-2 \cos \gamma \sin 2 \phi \sin 2 \psi\right\rangle,
$$

$$
G=-\frac{3}{2}\langle\sin 2 \gamma \cos \psi\rangle
$$

$$
H=\frac{1}{2}\langle\sin 2 \gamma \cos 2 \phi \cos \psi-2 \sin \gamma \sin 2 \phi \sin \psi\rangle .
$$


The four conventional order parameters $S, D, P$, and $C$ have originally been introduced by Straley [20]. One notes that our definition of $S, P$, and $C$ corresponds to the definition of the Straley parameters $S, T$, and $V$, respectively, while the expression for the order parameter $D$ contains an additional factor of $3 / 2$ compared with the definition of the order parameter $U$ used by Straley. See [36] for a relationship between different definitions of biaxial order parameters.

One also notes that the pseudovector order parameter $\mathbf{M}$ vanishes identically in the high-symmetry biaxial phase. Indeed, as any pseudovector, $\mathbf{M}$ changes sign under reflection with respect to a mirror plane which is parallel to it. In the high-symmetry biaxial nematic phase there are three orthogonal mirror planes, and thus all three components of $\mathbf{M}$ must vanish.

\section{B. Molecular theory and the parameters of the quadrupole potential}

Molecular-field theory is widely used in the description of uniaxial nematics [37], where the Maier-Saupe approach and its generalizations appear to combine simplicity and semiquantitative agreement with experimental data.

The molecular-field approach can readily be applied also to nematic liquid crystals composed of biaxial molecules and exhibiting the biaxial nematic phase. In this approximation, the free energy of the nematic phase reads

$$
\begin{aligned}
F / V= & \frac{1}{2} \rho^{2} \int f_{1}\left(\mathbf{a}_{1}, \mathbf{b}_{1}\right) u\left(\mathbf{r}, \mathbf{a}_{1}, \mathbf{a}_{2}, \mathbf{b}_{1}, \mathbf{b}_{2}\right) \\
& \times f_{1}\left(\mathbf{a}_{2}, \mathbf{b}_{2}\right) d \mathbf{r} d \mathbf{a}_{1} d \mathbf{b}_{1} d \mathbf{a}_{2} d \mathbf{b}_{2} \\
& +\rho k_{B} T \int f_{1}\left(\mathbf{a}_{1}, \mathbf{b}_{1}\right) \ln f_{1}\left(\mathbf{a}_{1}, \mathbf{b}_{1}\right) d \mathbf{a}_{1} d \mathbf{b}_{1},
\end{aligned}
$$

where $\rho$ is the molecular number density and $f_{1}\left(\mathbf{a}_{1}, \mathbf{b}_{1}\right)$ is the orientational distribution function which depends on the orientation of both long and short molecular axes with respect to the macroscopic directors.

The equilibrium distribution function is obtained by minimization of the free energy which yields the following expression:

$$
f_{1}(\mathbf{a}, \mathbf{b})=\frac{1}{Z} \exp \left[-\frac{U_{M F}(\mathbf{a}, \mathbf{b})}{k_{B} T}\right],
$$

where $Z$ is the normalization constant,

$$
Z=\int \exp \left[-\frac{U_{M F}(\mathbf{a}, \mathbf{b})}{k_{B} T}\right] d \mathbf{a} d \mathbf{b},
$$

and where the mean-field potential $U_{M F}$ is expressed as

$$
U_{M F}(\mathbf{a}, \mathbf{b})=\rho \int f_{1}\left(\mathbf{a}_{2}, \mathbf{b}_{2}\right) U_{\mathrm{eff}}\left(\mathbf{a}, \mathbf{a}_{2}, \mathbf{b}, \mathbf{b}_{2}\right) d \mathbf{a}_{2} d \mathbf{b}_{2} .
$$

Here $U_{\text {eff }}\left(\mathbf{a}_{1}, \mathbf{a}_{2}, \mathbf{b}_{1}, \mathbf{b}_{2}\right)$ is the effective interaction potential integrated over all intermolecular vectors $\mathbf{r}$ :

$$
U_{\mathrm{eff}}\left(\mathbf{a}_{1}, \mathbf{a}_{2}, \mathbf{b}_{1}, \mathbf{b}_{2}\right)=\int u\left(\mathbf{r}, \mathbf{a}_{1}, \mathbf{a}_{2}, \mathbf{b}_{1}, \mathbf{b}_{2}\right) d \mathbf{r} .
$$

In the general case the effective interaction potential $U_{\text {eff }}\left(\mathbf{a}_{1}, \mathbf{a}_{2}, \mathbf{b}_{1}, \mathbf{b}_{2}\right)$ depends on mutual orientation of the primary and secondary axes of the two interacting molecules. Fixing the orientation of one of the molecules one can express the potential as a function of the angles $\{\gamma, \phi, \psi\}$ which specify the orientation of the second molecule.

In the case of uniaxial molecules, the potential $U_{\text {eff }}$ depends only on the coupling between the primary axes of the molecules $\mathbf{a}_{1}$ and $\mathbf{a}_{2}$, i.e., $U_{\text {eff }}(1,2)=U_{\text {eff }}\left(\mathbf{a}_{1} \cdot \mathbf{a}_{2}\right)$. Such a potential can be expanded in Legendre polynomials $P_{n}\left(\mathbf{a}_{1} \cdot \mathbf{a}_{2}\right)$ and the first few terms of this expansion define a model interaction potential used in the Maier-Saupe theory and its generalizations [37]. A more complicated expansion of the effective interaction potential has also been employed in the molecular theory of smectics C [32].

For biaxial molecules, the general form of the potential $U_{\text {eff }}$ depends on the molecular symmetry. For molecules of the $C_{2 h}$ symmetry group, the potential can be expanded in powers of the three molecular tensors $Q_{i j}^{M}=a_{i} a_{j}-(1 / 3) \delta_{i j}$, $B_{i j}^{M}=b_{i} b_{j}-c_{i} c_{j}$, and $\Gamma_{i j}^{M}=b_{i} a_{j}+a_{i} b_{j}$ discussed in Sec. III A. Keeping the quadratic terms one obtains the following expression for the effective interaction potential:

$$
\begin{aligned}
U_{\mathrm{eff}}= & u_{1} \mathbf{Q}_{1}^{M}: \mathbf{Q}_{2}^{M}+u_{2} \mathbf{B}_{1}^{M}: \mathbf{B}_{2}^{M}+u_{3}\left(\mathbf{Q}_{1}^{M}: \mathbf{B}_{2}^{M}+\mathbf{B}_{1}^{M}: \mathbf{Q}_{2}^{M}\right) \\
& +u_{4} \boldsymbol{\Gamma}_{1}^{M}: \boldsymbol{\Gamma}_{2}^{M}+u_{5}\left(\mathbf{Q}_{1}^{M}: \boldsymbol{\Gamma}_{2}^{M}+\boldsymbol{\Gamma}_{1}^{M}: \mathbf{Q}_{2}^{M}\right)+u_{6}\left(\mathbf{B}_{1}^{M}: \boldsymbol{\Gamma}_{2}^{M}\right. \\
& \left.+\boldsymbol{\Gamma}_{1}^{M}: \mathbf{B}_{2}^{M}\right) .
\end{aligned}
$$

One notes that Eq. (22) defines the generalization of the socalled quadrupole interaction potential based on two molecular tensors which has been proposed by Straley [20] on semiphenomenological grounds and then used by other authors in the theory of the biaxial nematic phase [24,26,27].

Substituting the expressions for the unit vectors $\mathbf{a}, \mathbf{b}, \mathbf{c}$ [Eqs. (1)-(3)] one obtains the following expression for the potential:

$$
\begin{aligned}
U_{\mathrm{eff}}(\gamma, \phi, \psi)= & u_{1}\left(\cos ^{2} \gamma-1 / 3\right)+u_{2}\left[\operatorname { c o s } 2 \psi \operatorname { c o s } 2 \phi \left(\cos ^{2} \gamma\right.\right. \\
& +1)-2 \cos \gamma \sin 2 \phi \sin 2 \psi] \\
& +u_{3} \sin ^{2} \gamma(\cos 2 \psi+\cos 2 \phi) \\
& +u_{4}(2 \cos 2 \gamma \cos \phi \cos \psi \\
& -2 \cos \gamma \sin \phi \sin \psi)+u_{5} \sin 2 \gamma(\cos \phi \\
& -\cos \psi)+u_{6}[2 \sin \gamma(\sin \phi \sin 2 \psi \\
& -\sin 2 \phi \sin \psi)+\sin 2 \gamma(\cos 2 \phi \cos \psi \\
& -\cos 2 \psi \cos \phi)] .
\end{aligned}
$$

The combinations of trigonometric functions appearing in the right-hand side are mutually orthogonal, and this property can be used to present an arbitrary interaction potential, in the first approximation, as a linear combination of these functions. For a given interaction potential (e.g., for the interaction potential of a pair of model molecules) the coefficients $u_{1-6}$ of the effective quadrupole potential can be evaluated numerically by integrating the pair potential over the intermolecular vector and all relative orientations of the two molecules: 


$$
\begin{aligned}
& u_{1}=\frac{45}{32 \pi^{2}} \int_{0}^{\pi} d \gamma \sin \gamma \int_{0}^{2 \pi} d \phi \int_{0}^{2 \pi} d \psi U(\gamma, \phi, \psi) \\
& \times\left(\cos ^{2} \gamma-1 / 3\right) \text {, } \\
& u_{2}=\frac{5}{32 \pi^{2}} \int_{0}^{\pi} d \gamma \sin \gamma \int_{0}^{2 \pi} d \phi \int_{0}^{2 \pi} d \psi U(\gamma, \phi, \psi) \\
& \times\left[\cos 2 \psi \cos 2 \phi\left(\cos ^{2} \gamma+1\right)-2 \cos \gamma \sin 2 \phi \sin 2 \psi\right] \text {, } \\
& u_{3}=\frac{15}{64 \pi^{2}} \int_{0}^{\pi} d \gamma \sin \gamma \int_{0}^{2 \pi} d \phi \int_{0}^{2 \pi} d \psi U(\gamma, \phi, \psi) \\
& \times \sin ^{2} \gamma(\cos 2 \psi+\cos 2 \phi), \\
& u_{4}=\frac{5}{32 \pi^{2}} \int_{0}^{\pi} d \gamma \sin \gamma \int_{0}^{2 \pi} d \phi \int_{0}^{2 \pi}[d \psi U(\gamma, \phi, \psi)] \\
& \times(2 \cos 2 \gamma \cos \phi \cos \psi-2 \cos \gamma \sin \phi \sin \psi), \\
& u_{5}=\frac{15}{64 \pi^{2}} \int_{0}^{\pi} d \gamma \sin \gamma \int_{0}^{2 \pi} d \phi \int_{0}^{2 \pi} d \psi U(\gamma, \phi, \psi) \\
& \times \sin 2 \gamma(\cos \phi-\cos \psi) \text {, } \\
& u_{6}=\frac{5}{64 \pi^{2}} \int_{0}^{\pi} d \gamma \sin \gamma \int_{0}^{2 \pi} d \phi \int_{0}^{2 \pi} d \psi U(\gamma, \phi, \psi) \\
& \times[2 \sin \gamma(\sin \phi \sin 2 \psi-\sin 2 \phi \sin \psi) \\
& +\sin 2 \gamma(\cos 2 \phi \cos \psi-\cos 2 \psi \cos \phi)] \text {. }
\end{aligned}
$$

This effectively six-dimensional integration requires significant computational resources. We have performed the calculations for the potential [Eq. (4)] for a number of representative sets of parameters using the parallel processed MATLAB-STAR-P code at the Lonsdale cluster at Trinity Centre for High Performance Computing, Trinity College Dublin. In these calculations we have set $A=B=2 r_{0}$ (see Fig. 1). One notes that the increase in the ratio $A / B$ obviously leads to an increase in the elongation of the whole molecule which leads to an increase in the uniaxial coupling constant $u_{1}$ and thus to a decrease in the isotropic-nematic transition temperature.

We have studied the role of shape anisotropy (elongation) of the mesogenic groups, represented by the parameter $\kappa$, and the role of the tilt angle of the mesogenic groups with respect to the axes of the molecular frame on the coupling constants of the effective quadrupole potential. A typical example of the dependencies of the coupling constants $u_{1-6}$ on the parameter $\kappa$ is shown in Fig. 2 . One can readily see that the interaction constants $u_{4}, u_{5}$, and $u_{6}$ (shown by dashed lines), which determine the contributions from molecular biaxial tensors $\boldsymbol{\Gamma}_{1,2}^{M}$, are very small which indicates that the additional biaxial order parameters $G$ and $H$ should also be small. The dependence of the coupling constants on the tilt angle of the mesogenic groups is presented in Fig. 3. In this case, the constants $u_{4}$ and $u_{6}$ are also small and only the constant $u_{5}$ is slightly larger. One notes, however, that the

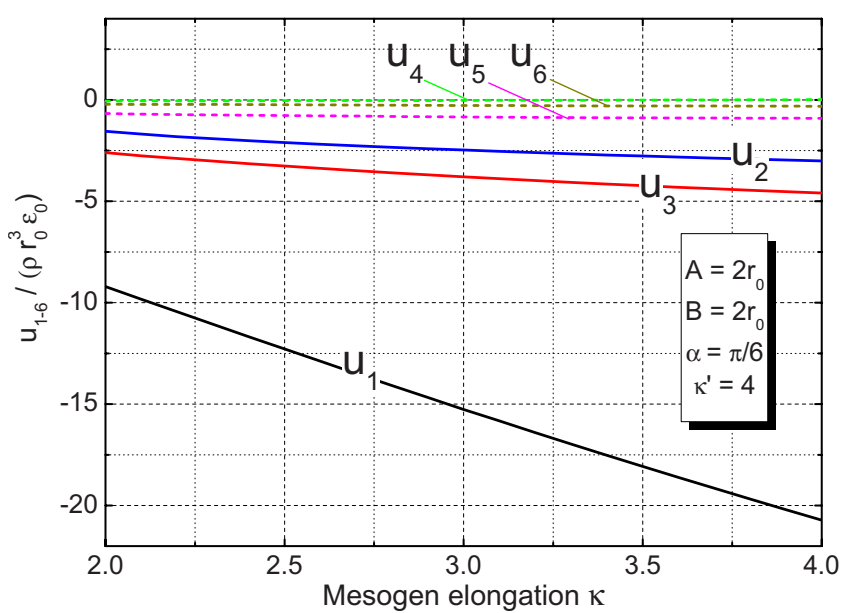

FIG. 2. (Color online) All dimensionless constants of the effective quadrupole potential $u_{1-6} /\left(\varepsilon_{0} r_{0}^{3} \rho\right)$ as functions of mesogen elongation $\kappa$ for $\alpha=\pi / 6, A=2 r_{0}, B=2 r_{0}$, and $\kappa^{\prime}=4$.

constant $u_{5}$ describes the coupling with the uniaxial molecular tensor $\mathbf{Q}^{M}$, and thus it is not expected to have a strong effect in the biaxial phase. At the same time, it is more important to note that the order parameters $G$ and $H$ vanish in the limit of $S \rightarrow 1$, i.e., in the case of perfect order of long molecular axes. Thus, at least far from the transition into the isotropic phase the order parameters $G$ and $H$ are expected to be small and for simplicity we will neglect them in the rest of this paper.

If the parameters $G$ and $H$ are not qualitatively important and the coupling constants $u_{4}, u_{5}, u_{6}$ are relatively small, the corresponding terms containing the tensor $\Gamma^{M}$ in the effective interaction potential can be neglected, and the relative stability of the biaxial nematic phase is determined by the ratios $u_{2} / u_{1}$ and $u_{3} / u_{1}$ where the constant $u_{1}$ mainly determines the isotropic-nematic transition temperature. Thus we now focus into the dependence of the constants $u_{1}, u_{2}, u_{3}$ on the parameters of the model.

One can readily see from Fig. 4 that the absolute values of the constants $u_{1}, u_{2}, u_{3}$ increase monotonically with the increasing elongation as one may expect. At the same time, the

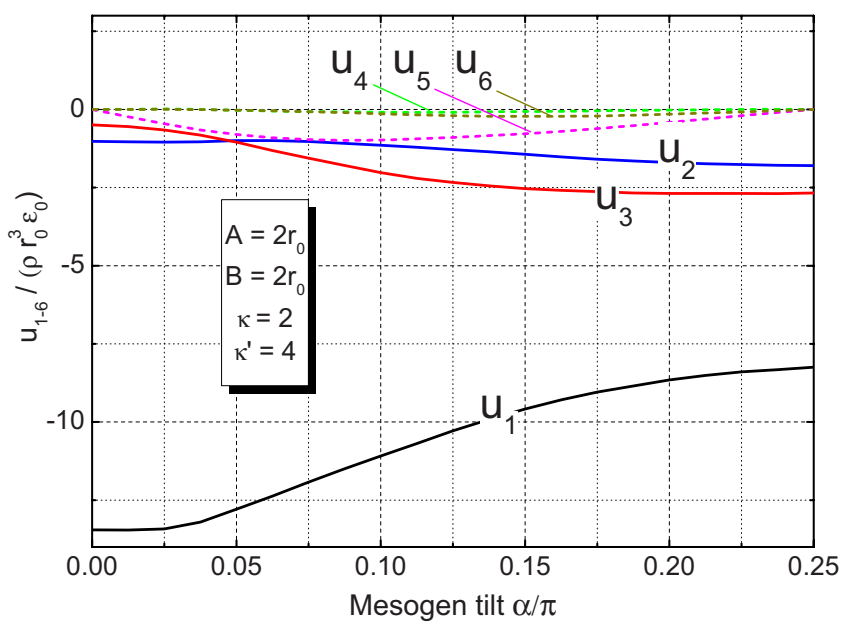

FIG. 3. (Color online) All dimensionless constants of the effective quadrupole potential $u_{1-6} /\left(\varepsilon_{0} r_{0}^{3} \rho\right)$ as functions of mesogen tilt angle $\alpha$ for $A=2 r_{0}, B=2 r_{0}, \kappa=2$, and $\kappa^{\prime}=4$. 


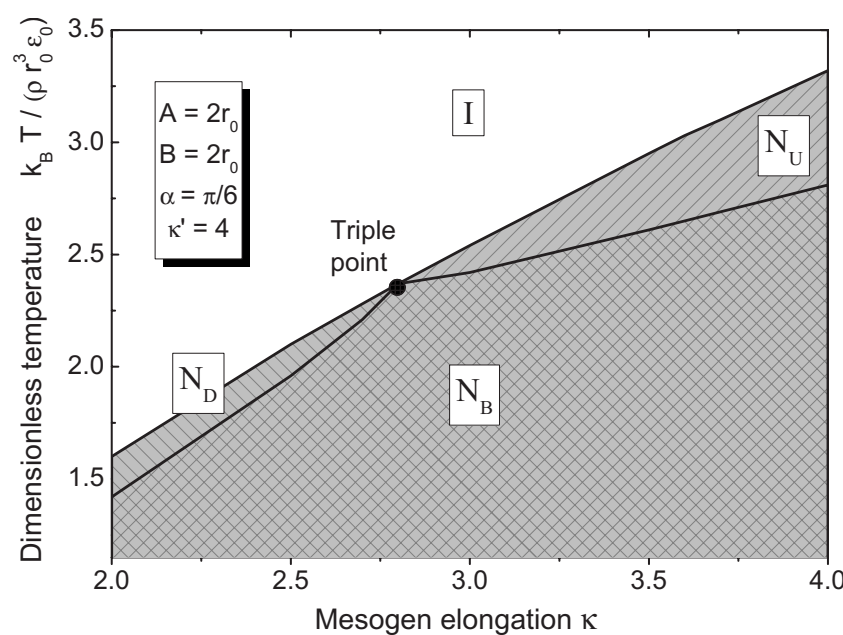

FIG. 4. Phase diagram corresponding to the constants of the mean-field potential from Fig. 2.

ratio of the constants $u_{2}$ and $u_{3}$ (which specify the biaxial part of the quadrupole interaction potential) to the constant $u_{1}$ (which determines the uniaxial interaction) weakly depends on $\kappa$. Thus an increase in the shape anisotropy of the mesogenic groups only weakly affects the ratio of the isotropic-uniaxial nematic and uniaxial nematic-biaxial nematic transition temperatures.

The dependence of the interaction constants in the meanfield potential on the tilt angle of the mesogenic groups with respect to the axes of the rectangular molecular frame is different as shown in Fig. 3. The absolute value of the constant $u_{1}$, responsible for the uniaxial nematic ordering, decreases with the increasing tilt angle, while the absolute values of the biaxial constants $u_{2}$ and $u_{3}$ increases slightly with the increasing tilt. This can be explained if one notices that the overall shape of our model molecule changes from a rectangle to a parallelogram upon tilting. This results in a broader and effectively less elongated distribution of the mesogenic groups, i.e., the molecule becomes more biaxial. It should be noted that the ratios $u_{2} / u_{1}$ and $u_{3} / u_{1}$ increase with the increasing tilt of the mesogenic groups leading to an increasing uniaxial-biaxial transition temperature and decreasing temperature interval of the uniaxial nematic phase. This can be a reason why the biaxial ordering has been observed in liquid crystals composed of tetrapode molecules of the $C_{2 h}$ symmetry and not in biaxial molecules of approximately rectangular shape.

\section{PHASE TRANSITIONS}

Substituting Eq. (23) into the general Eq. (20) and using Eqs. (11)-(14) for the order parameters $S, P, D$, and $C$ one obtains the following expression for the mean-field potential:

$$
\begin{aligned}
U_{M F}(\gamma, \phi, \psi)= & u_{1}\left[\frac{2}{3} S P_{2}(\cos \gamma)+\frac{1}{2} P \sin ^{2} \gamma \cos 2 \phi\right] \\
& +u_{2}\left\{D \sin ^{2} \gamma \cos 2 \psi+C[\cos 2 \phi \cos 2 \psi(1\right. \\
& \left.\left.\left.+\cos ^{2} \gamma\right)-2 \cos \gamma \sin 2 \phi \sin 2 \psi\right]\right\}
\end{aligned}
$$

$$
\begin{aligned}
& +u_{3}\left\{S \sin ^{2} \gamma \cos 2 \psi+\frac{2}{3} D P_{2}(\cos \gamma)\right. \\
& +\frac{1}{2} P\left[\cos 2 \phi \cos 2 \psi\left(1+\cos ^{2} \gamma\right)\right. \\
& \left.-2 \cos \gamma \sin 2 \phi \sin 2 \psi]+C \sin ^{2} \gamma \cos 2 \phi\right\} .
\end{aligned}
$$

Substituting now Eq. (25) into Eq. (18) and then into Eq. (17) one obtains the expression for the free energy of the biaxial nematic phase:

$$
\begin{aligned}
F / V= & -\frac{1}{2} \rho\left[u_{1}\left(\frac{2}{3} S^{2}+\frac{1}{2} P^{2}\right)+u_{2}\left(\frac{2}{3} D^{2}+2 C^{2}\right)\right. \\
& \left.+u_{3}\left(\frac{4}{3} S D+2 P C\right)\right]-\rho k_{B} T \ln Z,
\end{aligned}
$$

where the normalization factor $Z$ reads

$$
Z=\int_{0}^{\pi} \sin \gamma d \gamma \int_{0}^{2 \pi} d \phi \int_{0}^{2 \pi} d \psi \exp \left[-\frac{U_{M F}(\gamma, \phi, \psi)}{k_{B} T}\right] .
$$

Minimizing this free energy as a function of four scalar order parameters one can calculate the order parameters as functions of temperature for the biaxial and/or uniaxial nematic. We do this numerically by decreasing $T$ from highly ordered to lower ordered phases, i.e., studying the transitions upon cooling the liquid crystal. One notes that the simple minimization of the mean-field free energy is possible because the corresponding model interaction potential is fully attractive. In the case of a partially repulsive interaction potential one has to employ the so called minimax procedure (see $[26,38]$ ) when the free energy is minimized with respect to one group of order parameters and maximized with respect to other parameters.

One notices that the given definitions of the order parameters [Eqs. (11)-(14)] are not intrinsically symmetric with respect to the exchange of the molecular axes. Indeed, one has to select the primary (or "long") molecular axis a, and then the two remaining secondary axes are treated in a symmetric way. For biaxial molecules, however, it is not always obvious which axis is the primary one, i.e. which axis orders along the main director. In fact, by changing the parameters of the molecular structure one may also change the orientation of the primary axis within the molecular structure. For example, the primary axis of the biaxial ellipsoid with semiaxes $a, b$, and $c$ is parallel to the axis a if $a>b$ and $a>c$. However, if $a=b$ the ellipsoid becomes uniaxial and its primary axis is now parallel to the axis c. A similar change in the primary axis occurs also in the molecular model presented in Fig. 1 when one changes the anisotropy of the basic rectangular structure.

Fortunately, the general tensorial character of the molecular theory, employed in this paper, enables one to recalculate easily the order parameters related to different molecular frames. It is shown in the Appendix that if the axis $\mathbf{c}$ is 
chosen to be the primary one, the corresponding tensor order parameters, which can now be expressed in the form $Q_{i j}^{c}$ $=\left\langle c_{i} c_{j}-(1 / 3) \delta_{i j}\right\rangle$ and $B_{i j}^{c}=\left\langle a_{i} a_{j}-b_{i} b_{j}\right\rangle$, have the following diagonal representations similar to Eqs. (8) and (9):

$$
\begin{gathered}
Q_{i j}^{c}=S^{c}\left(h_{i} h_{j}-\frac{1}{3} \delta_{i j}\right)+\frac{1}{2} P^{c}\left(n_{i} n_{j}-m_{i} m_{j}\right), \\
B_{i j}=D^{c}\left(h_{i} h_{j}-\frac{1}{3} \delta_{i j}\right)+C^{c}\left(n_{i} n_{j}-m_{i} m_{j}\right),
\end{gathered}
$$

where the scalar order parameters are expressed as

$$
\begin{aligned}
S_{c} & =\frac{1}{4} S+\frac{3}{4} P+\frac{1}{4} D+\frac{3}{4} C, \\
P_{c} & =-\frac{1}{4} S+\frac{1}{8} P-\frac{1}{4} D+\frac{1}{4} C, \\
D_{c} & =-\frac{3}{4} S-\frac{9}{8} P+\frac{1}{4} D+\frac{3}{4} C, \\
C_{c} & =\frac{3}{4} S-\frac{3}{8} P-\frac{1}{4} D+\frac{1}{4} C .
\end{aligned}
$$

Therefore, it is not crucial which molecular frame is used in the following mean-field calculations. One can readily transform the results between the frames, find the physically meaningful one, and define the primary nematic order parameter $S$ accordingly.

One notes that if the coupling constants $u_{4}, u_{5}, u_{6}$ are small, the effective intermolecular interaction potential possesses the $D_{2 h}$ symmetry in the first approximation. It follows from this symmetry that the primary molecular axis must be parallel to one of the axes $\mathbf{a}, \mathbf{b}, \mathbf{c}$. If the last three terms in the effective interaction potential [Eq. (23)] are taken into account, the primary molecular axes may deviate from the axis a slightly. This deviation, however, is expected to be very small for small $u_{4}, u_{5}, u_{6} \ll u_{1}$ and can be calculated only in the context of a more general theory based on all three tensor order parameters.

We have performed the numerical calculations for the sets of parameters $u_{1-3}$ presented in Figs. 2 and 3 and obtained the phase diagrams shown in Figs. 4 and 5, respectively.

The qualitative structure of the phase diagrams is similar to the one obtained by the previous authors (see, for example, $[20,24])$ using a phenomenological quadrupole potential. In all cases the biaxial nematic phase separates two different uniaxial nematic phases which can be called calamitic and discotic nematic phases, respectively. There exists also a "triple" point where all three different nematic phases are in equilibrium with each other and with the isotropic phase. Numerical calculations indicate that in a rather broad vicinity of the triple point the discontinuity of the isotropic-uniaxial nematic phase transition is very small or virtually absent. This apparently originates from the growing molecular biaxiality associated with the increasing order parameter $D$. It should also be noted that at the triple point the intermolecular interaction potential is characterized by the higher $D_{4 h}$

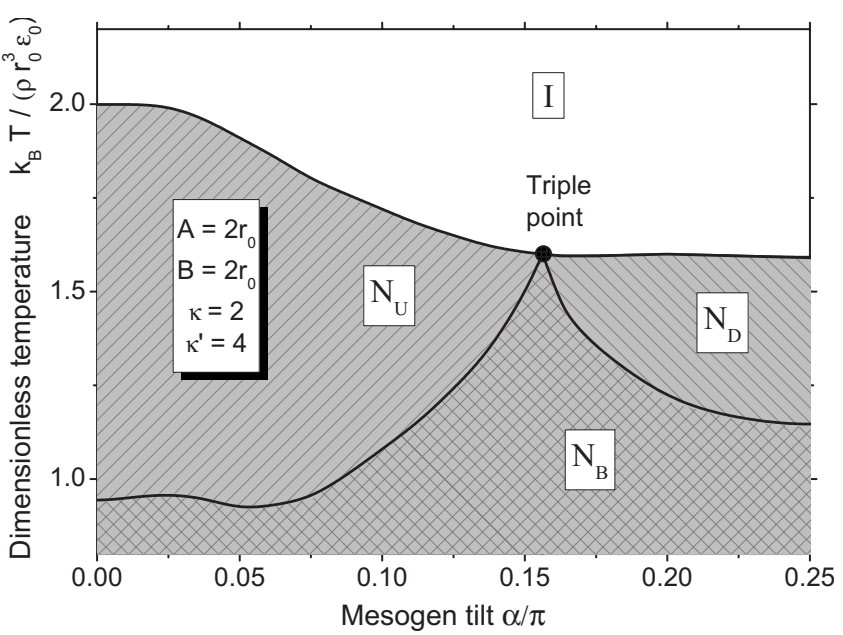

FIG. 5. Phase diagram corresponding to the constants of the mean-field potential from Fig. 3 .

symmetry [26], and as a result the system undergoes the direct second-order transition from the isotropic to the biaxial nematic phase. Thus in the vicinity of the triple point the discontinuity of the isotropic-nematic phase transition is indeed expected to be small.

It is interesting to consider the influence of the novel order parameters $G$ and $H$ on the triple point. As discussed above, the parameters $G$ and $H$ are expected to be small far from the isotropic-nematic transition. At the same time, in the vicinity of the triple point, all order parameters are small, and $G$ and $H$ should be taken into consideration. As shown in [26], at the triple point the primary molecular axis a is equivalent to one of the short axes, i.e., the conventional interaction potential (with $u_{4}=u_{5}=u_{6}=0$ ) is invariant under the exchange of these axes. In the case of the flat molecules considered in this paper the primary molecular axis a is equivalent to the axis $\mathbf{b}$ at the triple point. Then the additional tensor order parameter $\mathbf{G}$ does not have a direct effect on the triple point because the tensor $\mathbf{G}$ is invariant under the exchange of the axes $\mathbf{a}$ and $\mathbf{b}$ itself. In the general case the behavior may be more complicated and deserves a separate consideration.

The thermodynamic behavior resembles a conventional picture of the phase sequence in biaxial nematics: the liquid crystal first undergoes a first-order transition from the isotropic $(I)$ to the uniaxial nematic $\left(N_{U}\right)$ phase. Then at a lower temperature the second-order transition to the biaxial nematic $\left(N_{B}\right)$ phase occurs. Throughout the whole temperature range the order parameters $P$ and $D$ remain much smaller than $S$ and $C$. Typical examples are shown in Figs. 6(a) and 7(a).

After the triple point, the transition from the isotropic to another uniaxial nematic phase takes place. This uniaxial phase $N_{D}$ is ordered in a different way as the molecular axes c are now aligned along the main director. This phase is usually called the discotic nematic phase, and in such a phase, the planes of the molecules are aligned, which is equivalent to the alignment of the c-axes normal to those molecular planes. Accordingly, we present the results in terms of the c-related order parameters. 

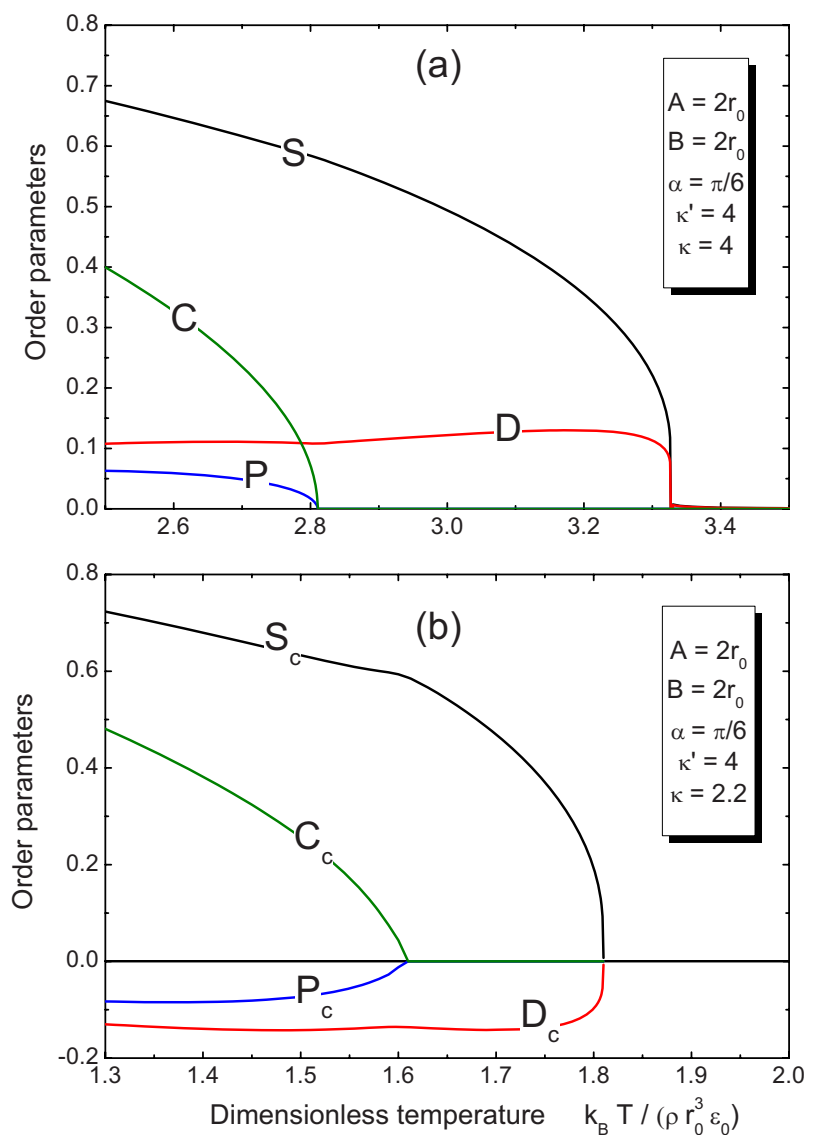

FIG. 6. (Color online) Temperature dependencies of the order parameters corresponding to the phase diagram depicted in Fig. 4 for (a) $\kappa=4$ and (b) $\kappa=2$.

One can readily see from Figs. 6(b) and 7(b) that below the transition from the isotropic phase, the liquid crystal is actually a uniaxial nematic. The tensor order parameters remain uniaxial around the $h$ axis and the only difference here from conventional nematic is the negative sign of $D_{c}$. At lower temperatures, a second-order transition to the biaxial nematic occurs. Qualitatively, the order parameters in the $N_{B}$ phase look rather similar in both $\mathbf{a}$ and $\mathbf{c}$ representations. The order parameters $S$ and $C$ are large, while $P$ and $D$ are relatively small and negative in the $\mathbf{c}$ representation. In other words, the phases $N_{D}$ and $N_{U}$, presented in the phase diagrams, are both uniaxial nematic phases composed of molecules of the same general structure, but the principal molecular axes are different. In the $N_{U}$ nematic phase the principal molecular axis is the axis a which is parallel to the long axes of the mesogenic groups, while in the $N_{D}$ phase the principal molecular axis is the axis $\mathbf{c}$ which is perpendicular to the molecular plane.

\section{DISCUSSION}

In this paper we have considered a model of a biaxial molecule of $C_{2 h}$ symmetry composed of four parallel mesogenic groups linked to the same center and located at the corners of a rigid rectangle. In the context of this model the molecular anisotropy can be controlled by changing the an-
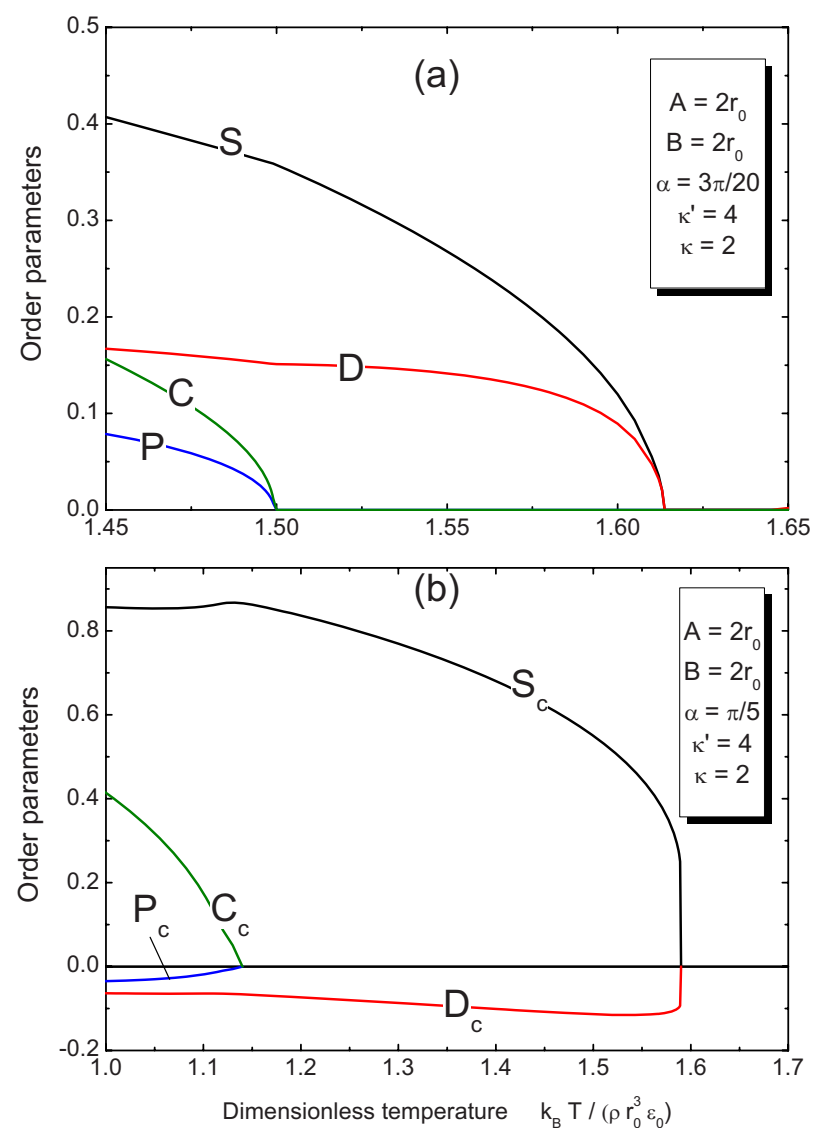

FIG. 7. (Color online) Temperature dependencies of the order parameters corresponding to the phase diagram depicted in Fig. 5 for (a) $\alpha=3 \pi / 20$ and (b) $\alpha=\pi / 5$.

isotropy of the mesogenic groups and/or the tilt angle of the mesogenic groups with respect to the axis of the rectangle. The interaction energy between two such molecules is taken to be equal to the sum of all pair interaction potentials of the mesogenic groups within the two molecules, and the interaction between the mesogenic groups is modeled by a standard Gay-Berne potential.

The total interaction potential between two such molecules has been expanded in orthogonal functions and approximately reduced to the so-called quadrupole effective interaction potential which has been proposed by Straley on semiphenomenological grounds and which is used in the existing molecular theory of biaxial nematics. The explicit form of the intermolecular interaction potential, based on a model for a biaxial molecule of the $C_{2 h}$ symmetry, enables one to calculate numerically the coupling constants of the effective quadrupole potential and to study their dependence on the parameters of the model molecular structure which has not been achieved before. All coupling constants have been calculated numerically as functions of the shape anisotropy (elongation) of the mesogenic groups and the tilt angle of the groups.

The increase in the elongation of the mesogenic groups results in the increase in the absolute values of both the constant $u_{1}$, which determined the uniaxial intermolecular interaction, and the constants $u_{2}$ and $u_{3}$, which specify the biaxial part of the interaction potential. As a result the relative 
strength of the biaxial interaction only weakly depends on the elongation and the biaxial nematic phase is not promoted. In contrast, the increase in the tilt angle of the mesogenic groups results in the decrease in the absolute value of the constant $u_{1}$ while the absolute values of the biaxial constants $u_{2}$ and $u_{3}$ slightly increase. This leads to an increase in the relative strength of the biaxial part of the total intermolecular interaction and thus to the increasing stability of the biaxial nematic phase.

This result enables one to speculate why the biaxial nematic phase has been discovered empirically in liquid crystals composed of tetrapode molecules which are characterized, on average, by the $C_{2 h}$ symmetry, rather than in various materials composed of strongly biaxial molecules of higher symmetry which have been synthesized and studied for a decade. Calculations presented in this paper indicate that in the context of the present molecular model the relationship between the uniaxial and biaxial parts of the effective intermolecular interaction potential can be controlled by the tilt of the mesogenic groups, and the relative strength of the biaxial interaction increases with the increasing tilt enhancing the stability of the biaxial nematic phase. One notes that in this paper we have used the simplified rigid model while real tetrapode molecules are flexible. Recently the role of flexibility in biaxial nematics has been taken into account [39] although it is still unclear how to obtain the parameters of the effective interaction potential for flexible molecules.

The effective interaction potential with numerically calculated coefficients has been used in the molecular-field theory of biaxial nematics to determine the order parameters and the phase diagrams of the system of biaxial molecules of $C_{2 h}$ symmetry. Orientational order parameters of the uniaxial and the biaxial nematic phases have been calculated numerically by direct minimization of the free energy for representative sets of the molecular model parameters. Two phase diagrams have been obtained enabling one to study the dependence of the transition temperatures on the shape anisotropy and the tilt angle of the mesogenic groups.

Both phase diagrams contain a triple point where all three nematic phases (uniaxial calamitic nematic, uniaxial discotic nematic, and biaxial nematic) are in equilibrium with the isotropic phase. Remarkably, the calamitic and discotic uniaxial nematic phases, presented in the phase diagrams, are composed of biaxial molecules of the same general structure but with different effective primary molecular axes. In the calamitic nematic phase, the primary molecular axes are parallel to the long axes of the mesogenic groups, while in the discotic nematic phase, the primary axes are perpendicular to the molecular planes. This reorientation of the primary molecular axis is related to the fact that the molecule becomes more disklike with the increasing tilt of the mesogenic groups. Statistically, both uniaxial phases, calamitic and discotic, are rather similar. The order parameters defined for the corresponding primary molecular axes exhibit similar temperature variations except for the sign of the order parameter $D$ which is negative in the discotic phase.

We note finally that the order parameters $P$ and $D$ appear to be smaller than the primary biaxial order parameter $C$ everywhere in the biaxial nematic phase for all sets of parameters. The same result has recently been obtained also in
[27] using one set of coupling constants, and relationship between the order parameters has also been confirmed by computer simulations [10]. As discussed in [27], this result differs qualitatively from the results obtained experimentally using the Fourier transform infrared spectroscopy [16]. Our preliminary analysis of the theory of IR absorption in biaxial nematics indicates that in one of the experimental geometries used in [16] the light wave in the medium is not transversal (due to birefringence), and the electric field is testing a different component of the averaged molecular IR polarizability. This, in principle, may lead to dramatic changes of the values of order parameters. The corresponding detailed analysis will be published elsewhere.

\section{ACKNOWLEDGMENTS}

The work was supported by EU BIND project No. FP7 216025, and SFI projects RFP 06/ENE039 and 07/W.1/ I1833. We are also grateful to the Trinity Centre for High Performance Computing (TCHPC) for the use of Lonsdale supercomputer. M.O. and M.G. are grateful to Epifanio Virga and Fulvio Bisi for valuable discussions and comments.

\section{APPENDIX: TRANSFORMATION OF THE ORDER PARAMETERS BETWEEN DIFFERENT MOLECULAR FRAMES}

If one chooses the molecular axis a as the primary one, the tensorial order parameters are conventionally defined as

$$
Q_{i j}=\left\langle a_{i} a_{j}-(1 / 3) \delta_{i j}\right\rangle, \quad B_{i j}=\left\langle b_{i} b_{j}-c_{i} c_{j}\right\rangle .
$$

If another molecular axis, say, the axis c, is supposed to be the primary one, a different pair of tensors is to be considered:

$$
Q_{i j}^{c}=\left\langle c_{i} c_{j}-(1 / 3) \delta_{i j}\right\rangle, \quad B_{i j}^{c}=\left\langle a_{i} a_{j}-b_{i} b_{j}\right\rangle .
$$

Using the identity $a_{i} a_{j}+b_{i} b_{j}+c_{i} c_{j}=\delta_{i j}$, one can easily find that

$$
Q_{i j}^{c}=-\frac{1}{2}\left(Q_{i j}+B_{i j}\right), \quad B_{i j}^{c}=\frac{3}{2} Q_{i j}-\frac{1}{2} B_{i j} .
$$

Next, using the identity $n_{i} n_{j}+m_{i} m_{j}+h_{i} h_{j}=\delta_{i j}$ one can rewrite the tensors [Eqs. (8) and (9)] as

$$
\begin{gathered}
Q_{i j}=\left(-\frac{1}{2} S-\frac{3}{4} P\right)\left(h_{i} h_{j}-\frac{1}{3} \delta_{i j}\right)+\frac{1}{2}\left(S-\frac{1}{2} P\right)\left(n_{i} n_{j}-m_{i} m_{j}\right), \\
B_{i j}=\left(-\frac{3}{2} C-\frac{1}{2} D\right)\left(h_{i} h_{j}-\frac{1}{3} \delta_{i j}\right) \\
+\left(-\frac{1}{2} C+\frac{1}{2} D\right)\left(n_{i} n_{j}-m_{i} m_{j}\right) .
\end{gathered}
$$

By substituting these tensors in such form in Eq. (A3) and collecting the terms proportional to $\left(h_{i} h_{j}-\frac{1}{3} \delta_{i j}\right)$ and $\left(n_{i} n_{j}\right.$ $-m_{i} m_{j}$ ) we finally arrive at Eqs. (28) and (29) with the scalar order parameters given by Eqs. (30)-(33). 
[1] M. J. Freiser, Phys. Rev. Lett. 24, 1041 (1970).

[2] L. J. Yu and A. Saupe, Phys. Rev. Lett. 45, 1000 (1980).

[3] G. R. Luckhurst and S. Romano, Mol. Phys. 40, 129 (1980).

[4] F. Biscarini, C. Chiccoli, P. Pasini, F. Semeria, and C. Zannoni, Phys. Rev. Lett. 75, 1803 (1995).

[5] M. P. Allen, Liq. Cryst. 8, 499 (1990).

[6] R. Berardi and C. Zannoni, J. Chem. Phys. 113, 5971 (2000).

[7] R. Berardi, C. Fava, and C. Zannoni, Chem. Phys. Lett. 236, 462 (1995).

[8] S. Sarman, Phys. Chem. Chem. Phys. 2, 3831 (2000).

[9] J. Pelaez and M. R. Wilson, Phys. Rev. Lett. 97, 267801 (2006).

[10] R. Berardi, L. Muccoli, S. Orlandi, M. Ricci, and C. Zannoni, J. Phys.: Condens. Matter 20, 463101 (2008).

[11] B. R. Acharya, A. Primak, and S. Kumar, Phys. Rev. Lett. 92, 145506 (2004).

[12] L. A. Madsen, T. J. Dingemans, M. Nakata, and E. T. Samulski, Phys. Rev. Lett. 92, 145505 (2004).

[13] B. R. Acharya, S.-W. Kang, and S. Kumar, Liq. Cryst. 35, 109 (2008).

[14] K. J. K. Semmler, T. J. Dingemans, and E. T. Samulski, Liq. Cryst. 24, 799 (1998).

[15] C. D. Southern, P. D. Brimicombe, S. D. Siemianowski, S. Jaradat, N. Roberts, V. Gortz, J. W. Goodby, and H. F. Gleeson, Europhys. Lett. 82, 56001 (2008).

[16] K. Merkel, A. Kocot, J. K. Vij, R. Korlacki, G. H. Mehl, and T. Meyer, Phys. Rev. Lett. 93, 237801 (2004).

[17] K. Merkel, A. Kocot, J. K. Vij, G. H. Mehl, and T. Meyer, J. Chem. Phys. 121, 5012 (2004).

[18] K. Neupane, S. W. Kang, S. Sharma, D. Carney, T. Meyer, G. H. Mehl, D. W. Allender, S. Kumar, and S. Sprunt, Phys. Rev. Lett. 97, 207802 (2006)

[19] J. L. Figueirinhas, C. Cruz, D. Filip, G. Feio, A. C. Ribeiro, Y. Frere, T. Meyer, and G. H. Mehl, Phys. Rev. Lett. 94, 107802 (2005).
[20] J. P. Straley, Phys. Rev. A 10, 1881 (1974).

[21] G. R. Luckhurst, C. Zannoni, P. L. Nordio, and U. Segre, Mol. Phys. 30, 1345 (1975).

[22] N. Boccara, R. Mejduni, and L. De Seze, J. Phys. (Paris) 38, 149 (1977).

[23] B. Mulder, Phys. Rev. A 39, 360 (1989).

[24] A. M. Sonnet, E. G. Virga, and G. E. Durand, Phys. Rev. E 67, 061701 (2003)

[25] G. De Matteis and E. G. Virga, Phys. Rev. E 71, 061703 (2005).

[26] F. Bisi, E. G. Virga, E. C. Gartland, G. De Matteis, A. M. Sonnet, and G. E. Durand, Phys. Rev. E 73, 051709 (2006).

[27] F. Bisi, G. R. Luckhurst, and E. G. Virga, Phys. Rev. E 78, 021710 (2008).

[28] D. W. Bruce, Chem. Rec. 4, 10 (2004).

[29] J. G. Gay and B. J. Berne, J. Chem. Phys. 74, 3316 (1981).

[30] E. de Miguel, L. F. Rull, M. K. Chalam, K. E. Gubbins, and F. Van Swol, Mol. Phys. 72, 593 (1991).

[31] P. I. C. Teixeira, M. A. Osipov, and G. R. Luckhurst, Phys. Rev. E 73, 061708 (2006).

[32] M. V. Gorkunov, M. A. Osipov, J. P. F. Lagerwall, and F. Giesselmann, Phys. Rev. E 76, 051706 (2007).

[33] C. Zannoni, J. Mater. Chem. 11, 2637 (2001).

[34] M. V. Gorkunov and M. A. Osipov, J. Phys. A: Math. Theor. 41, 295001 (2008).

[35] P. K. Karahaliou, A. G. Vanakaras, and D. J. Photinos, J. Chem. Phys. 131, 124516 (2009).

[36] R. Rosso, Liq. Cryst. 34, 737 (2007).

[37] M. A. Osipov, in Handbook of Liquid Crystals, 2nd ed., edited by D. Demus, J. Goodby, G. W. Gray, H.-W. Spiess, and V. Vill (Wiley-VCH, Weinheim, 1998), Vol. 1.

[38] E. C. Gartland and E. G. Virga, Arch. Ration. Mech. Anal. 196, 143 (2010).

[39] G. R. Luckhurst, Liq. Cryst. 36, 1295 (2009). 